\title{
Theoretical simulation of the influence of cathode formulation on lithium-ion battery performance
}

Cite as: AIP Conference Proceedings 2116, 250008 (2019); https://doi.org/10.1063/1.5114248

Published Online: 24 July 2019

D. Miranda, F. Miranda, A. M. Almeida, S. Lanceros-Méndez, and C. M. Costa

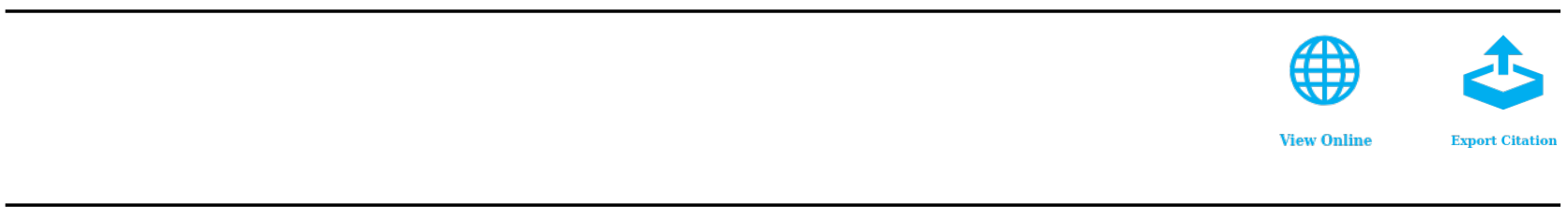

\section{Conference Proceedings}

Get $30 \%$ off all print proceedings!

\section{Enter Promotion Code PDF-30 at checkout}

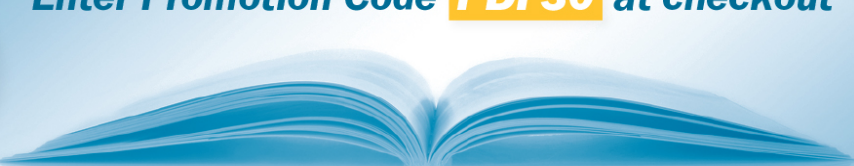




\title{
Theoretical Simulation of the Influence of Cathode Formulation on Lithium-Ion Battery Performance
}

\author{
D. Miranda ${ }^{1, a)}$, F. Miranda ${ }^{2,3, b)}$ A. M. Almeida $\left.4, \mathrm{c}\right)$, S. Lanceros-Méndez ${ }^{5,6, \mathrm{~d})}$ and C. \\ M. Costa ${ }^{4,7, e)}$ \\ '2Ai- Polytechnic Institute of Cávado and Ave, Barcelos, Portugal \\ ${ }^{2}$ CIDMA, Universidade de Aveiro, Aveiro, Portugal \\ ${ }^{3}$ Instituto Politécnico de Viana do Castelo, Viana do Castelo, Portugal \\ ${ }^{4}$ Centro de Física, Universidade do Minho, 4710-057 Braga, Portugal \\ ${ }^{5}$ BCMaterials, Basque Center for Materials, Applications and Nanostructures, UPV/EHU Science Park, 48940 \\ Leioa, Spain \\ ${ }^{6} I K E R B A S Q U E$, Basque Foundation for Science, Bilbao, Spain \\ ${ }^{7}$ Centro de Quimica, Universidade do Minho, 4710-057 Braga, Portugal \\ a)Corresponding author: damiranda@ipca.pt \\ b)fmiranda@estg.ipvc.pt \\ c)coimbra@fisica.uminho.pt \\ ${ }^{\mathrm{d})}$ senentxu.lanceros@bcmaterials.net \\ e)cmscosta@fisica.uminho.pt
}

\begin{abstract}
Optimizing cathode electrode formulation is essential in the development and performance of lithium-ion batteries, as the cathode affects the capacity of the battery. Cathode electrode formulation is based on different materials and relative contents. In this work, the cathode performance for the $\mathrm{LiMn}_{2} \mathrm{O}_{4}, \mathrm{LMO}$, active material has been obtained by theoretical simulations for different materials formulations and at various discharge rates. Further, the simulations were compared with experimental results. It is demonstrated that the optimization of the electrode formulation strongly depends on the percentage of conductive material, existing a minimum conductive filler content that optimizes the delivered capacity of the battery and being that delivery capacity independent of the conductive filler content for higher concentrations.
\end{abstract}

Keywords: Electrodes, simulation, capacity, lithium ion battery.

PACS: 88.80.F-, 88.80.ff, 88.05.-b

\section{INTRODUCTION}

Applications such as mobile phones, computers and electric vehicles use lithium-ion batteries [1]. Lithium-ion batteries are composed by anode (negative electrode), cathode (positive electrode) and separator/electrolyte and their performance can be improved through cathode formulation optimization [2].

Once the cathode influences the capacity of the battery and is constituted by different components (active material, binder and conductive additive), its optimization is essential to improve key issues of the battery such as specific energy and reliability, among others.

Considering the state-of-the art on cathode formulations, the highest reported amount of active material (C1) has been $95 \%$ and the lowest amount of conductive additive (C2) and binder (C3) were $3 \%$ and $2 \%$, respectively [3].

Theoretical simulations of lithium-ion batteries are essential tools to optimize various parameters such as electrodes, separators and geometries, which affect battery performance [4]. 
Taking into account that the cathode formulation has been optimized taking into consideration different active materials, binder and conductive additive relative contents, the goal of this work is to theoretically evaluate the influence of the relative content of the different materials of the cathode in cathode performance for the active material $\mathrm{LiMn}_{2} \mathrm{O}_{4}$, LMO.

This study focuses on the determination of the optimal relationship of the cathode components for obtain higher capacity, keeping the battery width constant, as illustrated in figure 1.

The Finite Element Method was used in all simulations by application of the Doyle/Fuller/Newmann model in two dimensions that describes the physical and electrochemical phenomena occurring during the operation of batteries [5].

The theoretical simulation for the different electrode compositions was validated through experimental results.

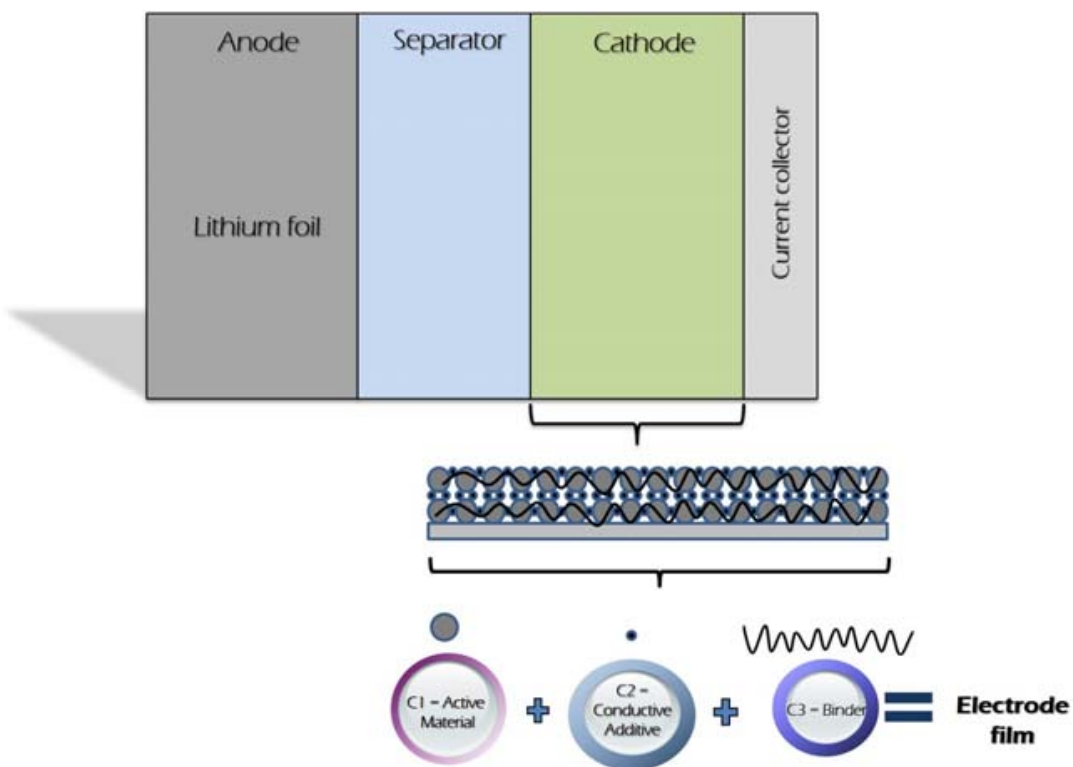

FIGURE 1. Lithium-ion battery geometry and cathode electrode composition.

Table 1 presents the main values of the different parameters used in the simulations for the separator and the cathode in $\mathrm{Li} / \mathrm{LiMn}_{2} \mathrm{O}_{4}$ half cells. The complete mathematical formulation of the simulations can be found in [4].

TABLE 1. Parameters used for the theoretical simulations of the Li/LMO half-cell.

\begin{tabular}{|c|c|c|c|}
\hline Parameter & Unit & Separator & Cathode $\left(\mathrm{Li}_{\mathbf{x}} \mathrm{Mn}_{2} \mathrm{O}_{4}\right)$ \\
\hline$C_{E, i, 0}$ & $\mathrm{~mol} / \mathrm{m}^{3}$ & & 3900 \\
\hline$C_{E, i, \max }$ & $\mathrm{mol} / \mathrm{m}^{3}$ & & 22860 \\
\hline$C_{L}$ & $\mathrm{~mol} / \mathrm{m}^{3}$ & 1000 & \\
\hline $\boldsymbol{R}$ & M & & $1.5 \times 10^{-6}$ \\
\hline$L_{i}$ & M & $430 \times 10^{-6}$ & $99 \times 10^{-6}$ \\
\hline $\boldsymbol{k}_{i}$ & $\mathrm{~S} / \mathrm{m}$ & $\left(\right.$ value $\left.^{\text {a) }}\right) \times 0,35^{1,5}$ & $\left(\right.$ value $\left.^{\mathrm{a})}\right) \times \varepsilon \mathrm{c}^{1,5}$ \\
\hline$D_{i}$ & $\mathrm{~m}^{2} / \mathrm{s}$ & $\left(7,5 \times 10^{-11}\right) \times 0,35^{1,5}$ & $7,5 \times 10^{-11} \times \varepsilon c^{1,5}$ \\
\hline$D_{L I}$ & $\mathrm{~m}^{2} / \mathrm{s}$ & & $1 \times 10^{-13}$ \\
\hline Brugg or p & & 8,5 & 1,5 \\
\hline$\varepsilon_{f, i}$ & & & 0,259 \\
\hline$\varepsilon_{i}$ & & 0,70 & \\
\hline$\tau$ & & 3,8 & \\
\hline$\sigma_{i}$ & $\mathrm{~S} / \mathrm{m}$ & & 3,8 \\
\hline$i_{1 C}$ & $\mathrm{~A} / \mathrm{m}^{2}$ & 17,5 & \\
\hline$T$ & $\mathrm{~K}$ & 298,15 & \\
\hline$A_{i}$ & $\mathrm{~m}^{2}$ & $1,8 \times 10^{-9}$ & $8,0 \times 10^{-8}$ \\
\hline$R$ & $\mathrm{~kg} / \mathrm{m}^{3}$ & 1008.98 & 2500 \\
\hline$V_{\text {Totalc }}$ & $\mathrm{m}^{3}$ & & $7,25 \times 10^{-9}$ \\
\hline $\boldsymbol{m}_{\text {Totalc }}$ & G & & $1,6 \times 10^{-3}$ \\
\hline
\end{tabular}

a) Model Fits: Ionic condutivity as a function of salt concentration in the $\mathrm{LiPF}_{6}$ in EC:DMC mixture [6] $k_{l}=2,905 \times 10^{-4}+2,32702 \times 10^{-2} c-1,82683 \times 10^{-2} c^{2}+5,1708 \times 10^{-3} c^{3}-4,977 \times 10^{-4} c^{4}$ 


\section{EXPERIMENTAL DETAILS}

The cathode film electrode was prepared by mixing LMO (C1), conductive additive (C2, Super P), and polymer binder (C3, PVDF) in NMP solvent with a weight ratio of 80:10:10 (wt.\%). After electrode preparation, Swagelok type cells were assembled in a home-made argon-filled glove box. The cells were composed by metallic lithium ( 8 $\mathrm{mm}$ diameter); Whatman glass microfiber filters (grade GF/A) (10 mm diameter) soaked in 1M LiPF6 in ethylene carbonate-dimethyl carbonate (EC-DMC, 1:1 vol) mixture and the prepared LMO electrode (8 $\mathrm{mm}$ diameter). Charge-discharge cycles were carried out at room temperature in the voltage range from 3.5 to $4.2 \mathrm{~V}$ at different scan rates $(\mathrm{C} / 10, \mathrm{C} / 5$ and $\mathrm{C} / 2)$ using a Landt CT2001A Instrument.

\section{RESULTS AND DISCUSSION}

Figure 2 shows the comparison of the experimental results and the simulation curves (red line) for Li/LMO halfcells at $\mathrm{C} / 10, \mathrm{C} / 5$ and $\mathrm{C} / 2$ discharge rate.

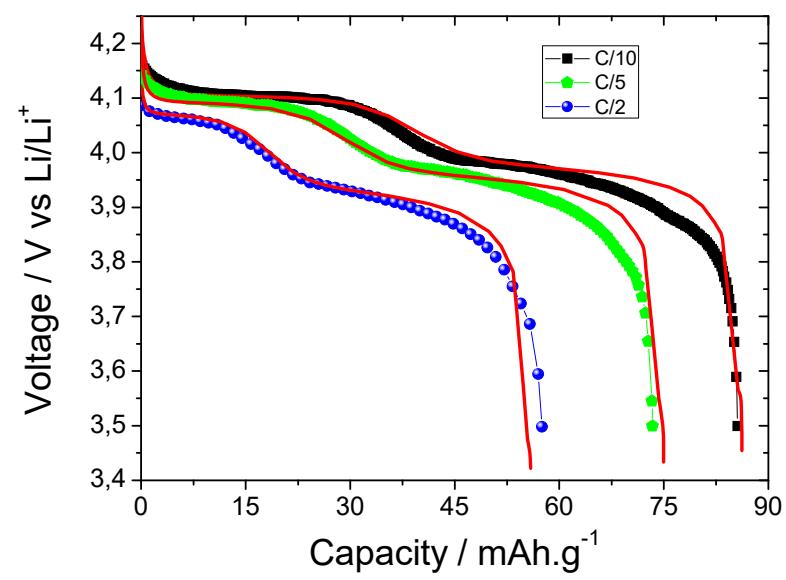

FIGURE 2. Voltage as a function of the delivered capacity at $\mathrm{C} / 10, \mathrm{C} / 5$ and $\mathrm{C} / 2$ discharge rates for the Li/LMO half-cells.

Regardless of discharge rate, figure 2 shows a good agreement between the theoretical values obtained in the simulations and the experimental curves, validating the theoretical model.

The slight difference between the experimental and theoretical curves is due to differences in the values of the theoretical and experimental electronic conductivity.

Figure 3a) shows the delivered capacity of the Li/LMO half-cells for different contents of active material (C1), from $50 \%$ until $95 \%$, with varying percentage of conductive additive (C2) and binder (C3).

Figure 3a) shows a different minimum percentage of $\mathrm{C} 2$ for each $\mathrm{C} 1$ content, after which a constant capacity value of the battery is obtained.

This percentage represent the minimum amount of conductive material required for obtain high battery performance due to the decrease of internal resistance during charge-discharge behavior.

In order to understand these phenomena, the Nyquist plots were simulated for the Li/LMO half-cells, as shown in figure $3 b$ ), for different relative contents of active material (C1), conductive additive (C2), and polymer binder.

Independently of the electrode composition, figure 3b) shows that the Nyquist plot is composed by a semicircle at high frequencies (overall resistance) and an inclined line in the low-frequency range, which can be considered as Warburg impedance. 

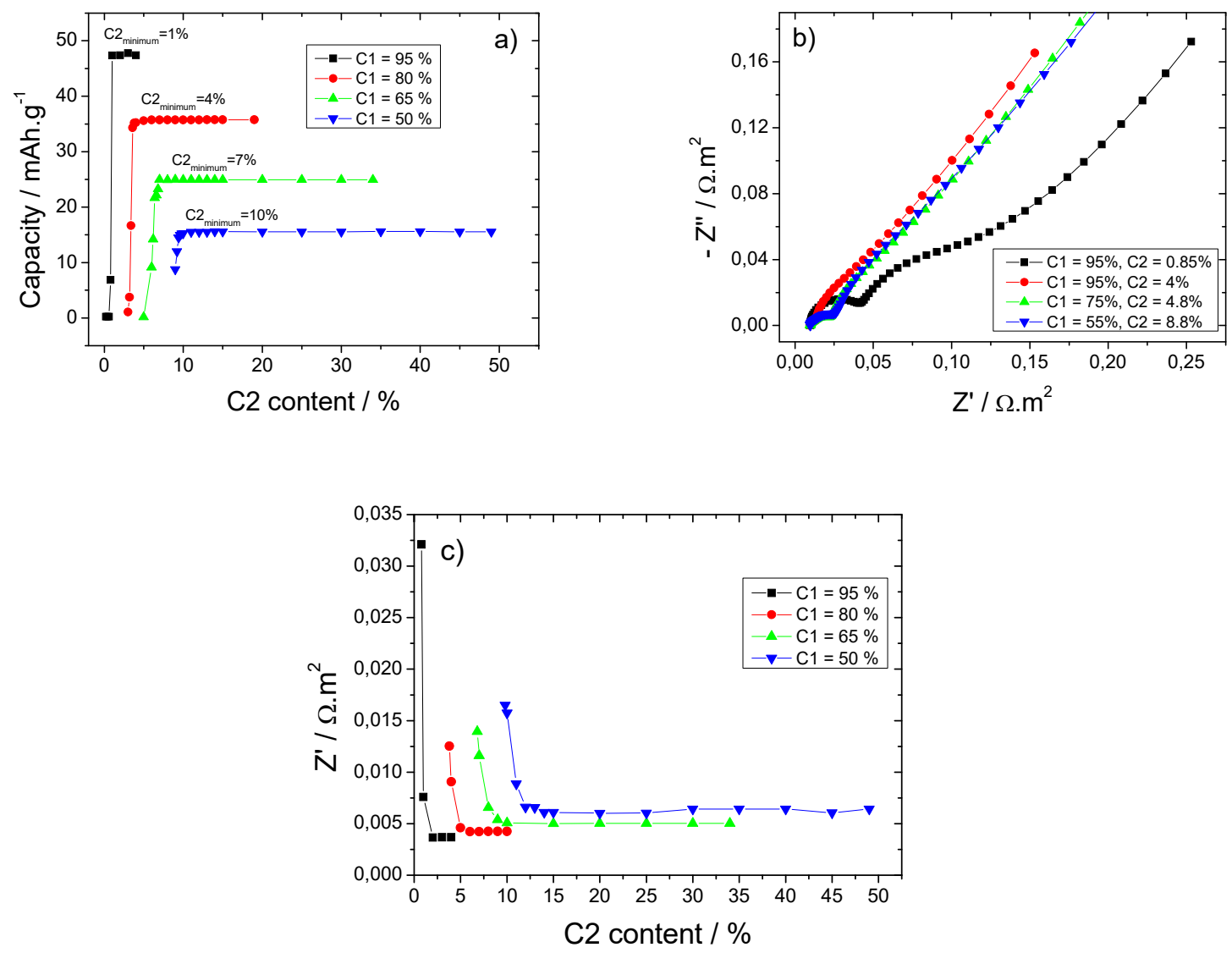

FIGURE 3. For Li/LMO half-cells: Delivered capacity with minimum of C2 (a), Nyquist plot (b) and total impedance as a function of $\mathrm{C} 2$ for different $\mathrm{C} 1$ at a discharge rate of $1 \mathrm{C}$.

Figure $3 \mathrm{~b}$ ) shows the Nyquist plot with $95 \%, 75 \%$ and $55 \%$ of active material $(\mathrm{C} 1)$, where it is observed that the impedance decreases with increasing conductive additive ( $\mathrm{C} 2)$ content when the $\mathrm{C} 1$ is constant. Figure 3c) shows that the impedance decreases with increasing conductive filler content, up to a critical concentration for each active material content, in which saturation occurs.

\section{CONCLUSION}

In order to improve lithium-ion battery performance, it is necessary the optimization of the electrode formulation based on different relative contents of active material, binder and conductive additive. In this work, cathode formulation is evaluated for $\mathrm{LiMn}_{2} \mathrm{O}_{4}, \mathrm{LMO}$, as active material, for different relative contents of active material, binder and conductive additive.

Further, the theoretical results were validated by experimental data.

The delivered capacity and Nyquist plot shows that optimization of the electrode formulation depends on the percentage of conductive material (C2) for different active materials content, where there is a minimum value of conductive material for obtaining high battery performance, being the delivery capacity independent of the conductive filler content, for higher conductive filler concentrations. 


\section{ACKNOWLEDGMENTS}

Portuguese Foundation for Science and Technology (FCT): UID/FIS/04650/2013, PTDC/CTM-ENE/5387/2014, UID/CTM/50025/2013 and grants SFRH/BPD/112547/2015 (C.M.C.), including FEDER funds through the COMPETE 2020 Programme and National Funds through FCT. Financial support from the Basque Government Industry Department under the ELKARTEK and HAZITEK programs is also acknowledged. F. Miranda was also supported by Portuguese funds through the CIDMA - Center for Research and Development in Mathematics and Applications, and the Portuguese Foundation for Science and Technology ("FCT - Fundação para a Ciência e a Tecnologia"), within project UID/MAT/04106/2013.

\section{REFERENCES}

1. M. Wakihara, O. Yamamoto, Lithium ion batteries: fundamentals and performance, Kodansha, 1998.

2. D. Miranda, et al., Applied Energy, 165 (2016) 318-328.

3. A Gören, et al., Composites Part B: Engineering, 83 (2015) 333-345.

4. D. Miranda, et al., Journal of Eletroanalytical Chemistry, 739 (2015) 97-110.

5. M. Doyle, et al., J. Electrochem. Soc. 143 (1996) 1890-1903.

6. J. W. Van Zee, et al., ECS Proceeding. 98-10 (1998) 360. 